\title{
Lactoferrin Coating Improves the Antibacterial and Osteogenic Properties of Alkali-Treated Titanium with Nanonetwork Structures
}

\author{
Derong Yin, ${ }^{1,2}$ Yonglong Hong $\left(\mathbb{D},{ }^{3}\right.$ Luyuan Chen $\mathbb{D},{ }^{2,4}$ Satoshi Komasa $\mathbb{D},{ }^{2}$ Yuanyuan Yang, ${ }^{2}$ \\ Honghao Zhang, ${ }^{2}$ Sifan Yan, ${ }^{2}$ Hiroshi Nishizaki $\mathbb{D}^{5}{ }^{5}$ Tetsuji Kusumoto, ${ }^{5}$ Wen Sui, ${ }^{2}$ \\ Takayoshi Kawazoe, 6 and Joji Okazaki $\mathbb{D}^{2}$ \\ ${ }^{1}$ State Key Laboratory of Oral Diseases and National Clinical Research Center for Oral Diseases, West China School of Stomatology, \\ Sichuan University, Chengdu 610041, China \\ ${ }^{2}$ Department of Removable Prosthodontics and Occlusion, Osaka Dental University, 8-1 Kuzuha-hanazono-cho, Hirakata, \\ Osaka 573-1121, Japan \\ ${ }^{3}$ Department of Oral and Maxillofacial Surgery, Shenzhen Hospital, Southern Medical University, 1333 Xinhu Road, Shenzhen, \\ 518100 Guangdong, China \\ ${ }^{4}$ Department of Stomatology Center, Shenzhen Hospital, Southern Medical University, 1333 Xinhu Road, Shenzhen, \\ 518100 Guangdong, China \\ ${ }^{5}$ Faculty of Health Sciences, Department of Oral Health Engineering, Osaka Dental University, 1-4-4 Makino-Honmachi, Hirakata, \\ Osaka 573-1121, Japan \\ ${ }^{6}$ Osaka Dental University, 8-1 Kuzuha-hanazono-cho, Hirakata, Osaka 573-1121, Japan
}

Correspondence should be addressed to Yonglong Hong; ylhong93@163.com

and Luyuan Chen; chen-luyuan900115@foxmail.com

Received 23 March 2020; Revised 24 June 2020; Accepted 6 July 2020; Published 28 August 2020

Academic Editor: Jianbo Yin

Copyright (C) 2020 Derong Yin et al. This is an open access article distributed under the Creative Commons Attribution License, which permits unrestricted use, distribution, and reproduction in any medium, provided the original work is properly cited.

Titanium and its alloys are the main dental implant materials used at present. The biological properties of pure titanium can be further improved by surface treatment methods. Alkali treatment of pure titanium at room temperature can form nanonetwork structures (TNS) on the surface, which has better osteoinductive ability than pure titanium. However, TNS does not possess antimicrobial properties, and bacterial infection is one of the main reasons for the failure of dental implant therapy. Therefore, it was the focus of our research to endow TNS with certain antimicrobial properties on the premise of maintaining its osteoinductive ability. Because of its excellent broad-spectrum antimicrobial properties and because it promotes osteoblast-like cell growth, lactoferrin (LF) was considered a promising prospect as a surface biological treatment material. In this study, bovine LF of physiological concentration was successfully coated on the surface of TNS to form the TNS-LF composite material. Results from in vitro and in vivo experiments showed that TNS-LF had better osteoinductive ability than TNS. Bacterial attachment and biofilm formation were also significantly decreased on the surface of TNS-LF. Therefore, this study has provided an experimental basis for the development of osteoinduction-antimicrobial composite implant materials for dental applications.

\section{Introduction}

With the increasing clinical use of dental implants and the expansion of indications for implant therapy, dental implants have become an important option for the treatment of patients with dentition defects and dentition loss [1-3]. This fact further highlights the need for scientific research towards promoting the continuous optimization of implant materials. Titanium and its alloy materials have become the main materials used for dental implantation surgery, as well as the focus of scientific researchers because of their suitable mechanical strength, biocompatibility, durability, nontoxicity, and 
other advantages. At present, the continuous scholarly indepth study of titanium and its alloy materials has revealed them as being increasingly multifunctional surfaces, that is, materials that can simultaneously respond to the colonization by different cells (osteoblasts, fibroblasts, vascular endothelial cells, etc.) and infectious factors (bacteria, etc.) in order to better adapt to their clinical applications, which have become the focus of research on titanium materials in recent years [4-6].

The different approaches used to produce multifunctional titanium surfaces include inorganic coating, functional organic coating treatment, and chemical surface treatment. However, researchers are progressively inclined to combine various surface functionalization methods to obtain better biological effects $[4,7]$. Kim et al. used alkali and heat treatment to improve the biological properties of these materials [8]. Because this treatment is relatively simple and inexpensive and might be able to increase the possibilities of clinical applications, many researchers have subsequently carried out in-depth research on it [7,9-17]. Through our research, we defined suitable conditions for forming a nanonetwork structure on the surface of titanium (TNS) using alkali treatment of titanium and its alloys at room temperature [10]. We subsequently analyzed the surface of TNS in detail and proved the superiority of this material on osteogenesis in both in vitro and in vivo experiments $[11,12]$. On this basis, we coated the TNS surface with fluorinated hydroxyapatite and amelogenin and the superior properties of these materials were experimentally verified $[13,14]$. On the contrary, it is undeniable that TNS does not possess antimicrobial properties. Therefore, we did some research and found that UV irradiation can effectively improve the antimicrobial properties of TNS $[15,16]$.

Lactoferrin (LF) is a multifunctional protein with many biological functions, such as antibacterial, anti-inflammatory, iron homeostasis, osteogenesis, angiogenesis, and immune regulation [18-26]. Iron homeostasis is essential for cell metabolism in vivo, and LF is well known for its great affinity for free iron [27]. As such, this iron binding ability might help regulate and alter iron metabolism during infection, inflammation, or other physiological functions [22].

In healthy subjects, lactoferrin circulates at a physiological concentration of $2-7 \mu \mathrm{g} / \mathrm{mL}$, and acts as a growth factor in inducing the growth and activity of osteoblasts, inhibiting the development of osteoclasts in vitro, and promoting bone growth in vivo [22, 28-32]. There have been numerous studies on the effects of different concentrations of LF on osteogenesis and vascularization, as well as on its antimicrobial activity. From these results, we could identify that for LF concentrations in the range of $1-100 \mu \mathrm{g} / \mathrm{mL}$, the osteogenic induction ability increased with the increase of LF concentration $[22,30,31,33,34]$. On that note, $8 \mu \mathrm{g} / \mathrm{mL}$ was the maximum concentration of LF not resulting in a negative impact on endothelial cell adhesion, whereas a higher concentration was used to inhibit vascularization [35]. Additionally, these LF concentrations were shown to play a certain role in the antibacterial properties of these materials $[20,21]$.

Therefore, in this experiment, in order to further optimize the osteogenic induction ability of TNS and increase its antimicrobial properties, we chose LF at a concentration of $10 \mu \mathrm{g} / \mathrm{mL}$ as the material for optimizing the biological properties of the TNS surface and formed a new TNS-LF implantation system. Subsequently, the surface properties of TNS and TNS-LF materials were analyzed in detail. The antimicrobial properties of both materials were analyzed using Staphylococcus aureus as an experimental model organism. In addition, the biological properties of these 2 materials were evaluated through cell and animal experiments. In this study, we hope to provide some experimental basis for future research and clinical applications of this simple and inexpensive surface treatment method used for the generation of pure titanium implants.

\section{Material and Methods}

2.1. Sample Preparation. Titanium disks (JIS 2 grade, diameter $15 \mathrm{~mm}$, thickness $1 \mathrm{~mm}$; Engineering Test Service, Osaka, Japan), sequentially polished with several grades of abrasive paper (Waterproof Paper ${ }^{\circledR}$ Nos. 800, 1000, and 1500; Riken Corundum Co. Ltd., Saitama, Japan), and titanium screw implants (external diameter $1.2 \mathrm{~mm}$, length $12 \mathrm{~mm}$; Nishimura Metal, Fukui, Japan) were used in this study. In order to reduce pollution on the surface of titanium disks and implants before alkali treatment, we ultrasonically cleaned samples for $10 \mathrm{~min}$ in a succession of acetone, ethanol, and deionized water. After the samples were completely dried, they were immersed in alkali solution $(10 \mathrm{M} \mathrm{NaOH})$ for $24 \mathrm{~h}$ at $30^{\circ} \mathrm{C}$ and then transferred into ultrapure water. Ultrapure water was replaced every $5 \mathrm{~min}$, until rinsate conductivity was $<5 \mu \mathrm{S} / \mathrm{cm}^{3}$, and subsequently, samples were removed and dried at room temperature $\left(20-28^{\circ} \mathrm{C}\right)$. After dry heat sterilization, the dried TNS material was used as a control in the following experiments. The obtained TNS was sterilized by dry heat, and the experimental steps thereafter were carried out in a sterile environment. Bovine LF (FUJIFILM Wako Pure Chemical Corporation, Osaka, Japan) concentration was adjusted by gradient dilution with deionized water to $10 \mu \mathrm{g} / \mathrm{mL}$, and $60 \mu \mathrm{l}$ of LF solutions was applied twice to the surface of TNS materials; the amount of protein coating on the surface of each sample is $0.6 \mu \mathrm{g}$. After completely drying, TNS-LF materials were used in subsequent experiments. The whole process guaranteed the stability of the operation and strictly abided by aseptic principles.

\subsection{Characterization of Surfaces}

2.2.1. Morphological Analysis. Surface morphology of TNS and TNS-LF samples were examined by scanning electron microscopy (SEM) (S-4800; Hitachi, Tokyo, Japan) at a magnification of $50.0 \mathrm{k}$ and $100.0 \mathrm{k}$ with an accelerating voltage of $5 \mathrm{kV}$, and 4 images were taken for each sample.

2.2.2. Surface Topography Analysis. Surface topography, mean average surface roughness $(\mathrm{Ra})$, and mean peak-tovalley height $(\mathrm{Rz})$ were examined using atomic force microscopy (AFM) (SPM-9600; Shimadzu, Tokyo, Japan). Scanning areas were approximately $2 \times 2 \mu \mathrm{m}$, and 4 different observation points were randomly selected for each sample. 
2.2.3. Physicochemical Property Analysis. Attenuated reflectance Fourier transform infrared spectroscopy (ATR-FTIR) was used to determine the physicochemical properties of samples at the range of $400-4000 \mathrm{~cm}^{-1}$.

2.2.4. Chemical Characterization (XPS). X-ray photoelectron spectrometry (XPS; PHI X-tool; ULVAC-PHI, Inc., Kanagawa, Japan) was used to analyze the chemical composition of the modified TNS and TNS-LF surface layers.

\subsection{Evaluation of Antimicrobial Properties}

2.3.1. Bacterial Cultivation. Staphylococcus aureus (ATCC 12600) was used in experiments evaluating the antimicrobial properties of samples. Two or three single colonies were picked and subsequently cultivated in trypticase soy broth with shaking overnight (16-18h). Fresh trypticase soy broth was then used to dilute the bacterial suspension and seeded on samples (TNS and TNS-LF disks) in standard 12-well polystyrene culture plates. Sterile water was added between wells to prevent the bacterial suspension from drying, and the bacterial suspension was then incubated aerobically at $37^{\circ} \mathrm{C}$.

2.3.2. Antibacterial Activity Assay. Twenty microliters of diluted bacterial suspension $\left(2.5 \times 10^{5} \mathrm{cfu} / \mathrm{mL}\right)$ was seeded on samples (TNS and TNS-LF disks), and following bacterial attachment to disks after a $24 \mathrm{~h}$ incubation, cultured bacteria were collected in $2 \mathrm{~mL}$ fresh broth using rapid vortex mixing for $3 \mathrm{~min}$. The recovered bacterial liquid was serially diluted to a certain concentration, introduced to trypticase soy agar culture plates, and cultured for another $24 \mathrm{~h}$. The number of colonies was counted, and the antimicrobial rate was calculated. The antimicrobial rate was calculated according to the following equation: $\%=(A-B) / A \times 100 \%$. Here, $A$ is the average number of bacteria on TNS (number of cfu), and $B$ is the average number of bacteria on TNS-LF (number of $\mathrm{cfu}$ ).

2.3.3. Analysis of Bacterial Biofilm Formation by Crystal Violet Staining. In order to detect biofilm formation, $2 \mathrm{~mL}$ of bacterial solution adjusted at $0.1 \mathrm{OD}$ was used on each sample material. After a $24 \mathrm{~h}$ incubation, samples were gently rinsed with phosphate buffer saline (PBS, nacalai tesque, Kyoto, Japan) and incubated with $2 \mathrm{~mL} 0.05 \% \mathrm{w} / \mathrm{v}$ crystal violet dye for $20 \mathrm{~min}$ at room temperature; then, residual dyes were removed by gently rinsing with PBS, transferred to a 24 -well plate, and destained in $1 \mathrm{~mL} 95 \%$ ethanol with rotary shaking for $20 \mathrm{~min}$ at room temperature. After destaining, $100 \mu \mathrm{L}$ of ethanol was transferred to each well and absorbance at $595 \mathrm{~nm}$ was measured on a SpectraMax M5 96-well microplate reader (Molecular Devices LLC, Sunnyvale, CA, USA).

2.3.4. Analysis of Initial Bacterial Attachment by Confocal Laser-Scanning Microscopy and Live/Dead Staining. As described previously, $20 \mu \mathrm{L}$ of diluted bacterial suspension $\left(2.5 \times 10^{5} \mathrm{cfu} / \mathrm{mL}\right)$ was seeded on samples (TNS and TNSLF disks). After incubation for 3 and $6 \mathrm{~h}$, bacterial suspension was removed, and discs were transferred to a new 24-well polystyrene culture plate. Unattached bacteria were removed by gently rinsing with sterile PBS and then stained for $15 \mathrm{~min}$ using the Live/Dead ${ }^{\circledR}$ BacLight $^{\mathrm{TM}}$ Bacterial Viability Kit (L7012) following the manufacturer's instructions. Subsequently, excess dyes were removed and samples placed on slides were immediately photographed under a confocal laser scanning microscope (LSM 700; Carl Zeiss, Jena, Germany).

2.3.5. Reactive Oxygen Species (ROS) Level Evaluation. Consecutively, $20 \mu \mathrm{L}$ of bacterial solution $\left(2.5 \times 10^{5} \mathrm{cfu} / \mathrm{mL}\right)$ was used to evaluate the level of reactive oxygen species (ROS). Following sample incubation for 3 and $6 \mathrm{~h}$, discs were gently washed with PBS and $300 \mu \mathrm{L}$ of $2^{\prime}, 7^{\prime}$-dichlorodihydrofluorescin diacetate $(10 \mu \mathrm{M}, \mathrm{DCFH}-\mathrm{DA}$; Sigma-Aldrich, St. Louis County, USA) was added to each disc surface and incubated for $30 \mathrm{~min}$ at $37^{\circ} \mathrm{C}$. After incubation, excess dye was washed away with PBS and samples were observed using a confocal laser-scanning microscope (LSM 700; Carl Zeiss, Jena, Germany).

\subsection{Osteogenic Characterization In Vitro}

2.4.1. Cell Culture. This study was conducted in accordance with the Osaka Dental University Animal Experiment Guide (Grant No. 18-03007). Both kinds of cells were cultured in a humidified atmosphere with $5 \% \mathrm{CO}_{2}$ at $37^{\circ} \mathrm{C}$, and medium was replaced every $3 \mathrm{~d}$. When cultures reached a confluence of $\sim 80 \%$ in $75 \mathrm{~cm}^{2}$ flasks, cell passaging was performed.

(1) Cell Culture of Rat Bone Marrow Mesenchymal Stem Cells (rBMMSCs). Rat bone marrow mesenchymal stem cells (rBMMSCs) were extracted from the femur of 8-week-old Sprague-Dawley rats (Shimizu Laboratory Supplies Co., Kyoto, Japan) and incubated in $75 \mathrm{~cm}^{2}$ flasks abiding by a previously described method [7]. Following incubation, well adherent-grown cells (third- and fourth cell generations) were digested by trypsin, and after digestion was stopped with added fresh medium, cell cultures were centrifuged, supernatant was discarded, cell pellets were resuspended in fresh medium, and cells were counted as $4 \times 10^{4}$ cells $/ \mathrm{mL}$ for subsequent in vitro experiments.

(2) Cell Culture of Rat Periodontal Ligament Cells (rPLCs). Rat periodontal ligament cells (rPLCs) were purchased from Lonza (Walkersville, MD, USA), and according to recommendations, medium (SCBM ${ }^{\mathrm{TM}}$ Stromal Cell Basal Medium, Lonza, Basel, Switzerland) was allocated at appropriate concentrations for cell culture. The methods of thawing, resuscitating, and subculturing of cells were reported in previous studies [14].

2.4.2. Cell Adhesion and Morphology. Cell adhesion was assessed using the Cell Titer-Blue ${ }^{\circledR}$ Cell Viability Assay (Promega, Madison, WI, USA) according to the manufacturer's protocol. Measurements of rBMMSC and rPLC cell adhesion were obtained at $1,3,6$, and $24 \mathrm{~h}$. Incubated TNS and TNSLF disks were washed twice with PBS and treated with $300 \mu \mathrm{L}$ diluted Cell Titer-Blue ${ }^{\circledR}$ Reagent (50 $\mu \mathrm{L}$ Cell TiterBlue $^{\circledR}$ Reagent diluted in $250 \mu \mathrm{L}$ PBS and prepared 
immediately before the PBS wash). After $1 \mathrm{~h}$ incubation, $100 \mu \mathrm{L}$ of the reagent from each well was added to a 96-well tissue culture plate and fluorescence was measured at $560 / 590 \mathrm{~nm}$ using a microplate reader (SpectraMax M5; Molecular Devices LLC, Sunnyvale, CA, USA). Measurements of rBMMSC and rPLC cell morphology were obtained at 3 and $6 \mathrm{~h}$. Confocal laser-scanning microscopy (LSM 700; Carl Zeiss, Jena, Germany) was used for cell examination, and the respective dyeing method was performed according to the previously described experimental method [7].

2.4.3. Detection of Cell Osteogenic Markers. Analysis of alkaline phosphatase (ALP) activity, quantification of calcium deposition in the extracellular matrix, osteocalcin (OCN) production, and expression levels of osteogenesis-related genes were used to detect the osteogenic differentiation induction ability of TNS and TNS-LF to rBMMSCs and rPLCs.

To evaluate induced ALP activity, rBMMSCs and rPLCs at a density of $4 \times 10^{4}$ cells/well were seeded on 24 -well plates containing TNS and TNS-MAP disks, respectively. After the medium was cultured for 1 week, it was replaced with differentiation-inducing medium [7]; thereafter, cells were cultured for an additional 7 and 14 days, and then, the ALP activity of cells on the surfaces of TNS and TNS-LF was analyzed. Medium was changed every 3 days. Details on the respective method have been previously described $[7,14]$. As described above, the differentiation-inducing medium was replaced after 1 week of cell culture, cells were further cultured for 21 or $28 \mathrm{~d}$, and consecutively, quantification of calcium deposition in the extracellular matrix and osteocalcin $(\mathrm{OCN})$ production of cells on the surfaces of TNS and TNS-LF were evaluated using the methods previously described [7].

To evaluate the expression levels of osteogenesis-related genes, total RNA of rBMMSCs and rPLCs cultured for 3, 7, 14, and $21 \mathrm{~d}$ on TNS and TNS-LF sample disks was isolated using the RNeasy Mini Kit (Qiagen, Venlo, The Netherlands) and a TaqMan real-time RT-PCR assay (Life Technologies, Carlsbad, CA, USA), as previously described [7]. The expression levels of runt-related transcription factor 2 (Runx2), $A L P$, bone morphogenetic protein $(B M P)$, and osteopontin $(O P N)$ were measured. The StepOnePlus ${ }^{\mathrm{TM}}$ Real-Time PCR System (Life Technologies) was used to quantify these osteogenesis-related genes. Relative gene expression levels of each group were determined using the $2^{-\Delta \Delta \mathrm{Ct}}$ method and normalized to that of the housekeeping gene glyceraldehyde 3-phosphate dehydrogenase (GAPDH) [7].

\subsection{Osteointegration Characterization In Vivo}

2.5.1. Animal Model and Surgical Procedures. As in our previous study, 10 male Sprague-Dawley rats aged 8 weeks (Shimizu Laboratory Supplies Co.) were randomly assigned to each of the TNS and TNS-LF groups. Previous reports have described in detail the experimental methods used, including the surgical procedure of sample implantation and postoperative care $[7,17]$. Animal studies were conducted in accordance with the National Institutes of Health guide and the ethical guidelines of the Animal Care and Use Committee of Osaka Dental University (Approval No. 18-03007).

2.5.2. Analysis of Animal Experimental Results. Microcomputed tomography (micro-CT) and histological preparation and histomorphometric assessment and analysis performed were consistent with methods previously used [7, 17]. The regions of interest (ROI; $500 \mu \mathrm{m}$ around the implant and $2 \mathrm{~mm}$ below the epiphyseal line) in the CT images, bone volume fraction (BV/TV, \%), trabecular number (Tb.N, $\mathrm{mm}^{-1}$ ), trabecular separation (Tb.Sp, $\mu \mathrm{m}$ ), and trabecular thickness (Tb.Th, $\mu \mathrm{m}$ ) were evaluated using the Morphometric software (TRI/3D-BON; Ratoc System Engineering, Tokyo, Japan). Bone area ratio (BA), boneimplant contact (BIC), and the area of new bone at 1,4 , and 8 weeks were also analyzed.

2.6. Statistical Analysis. Surface characterizations, bacterial experiments, cell experiments, and in vivo experiments were each conducted in triplicate. All quantitative results were expressed as means \pm standard deviations. Results were evaluated by one-way analysis of variance (ANOVA) and Bonferroni's post hoc test using SPSS 20.0 software (IBM Corporation, Armonk, NY, USA); $P<0.05$ was considered statistically significant.

\section{Results}

3.1. Characterization of Surfaces. The surface characteristics of the 2 materials in both the control and experimental groups were analyzed as shown in Figure 1, Table 1, and Table 2. Figure 1(a) presents SEM images of the surface morphology of the 2 kinds of materials at different magnifications. Under $50 \mathrm{k}$ and $100 \mathrm{k}$ magnification (especially at $\times 100 \mathrm{k}$ ), it can be clearly seen that the surface of the TNS material has formed a uniform nanonetwork structure, and this nanonetwork is relatively sharp and slender. Although the nanonetwork was obviously thicker and rounder in the TNS-LF surface, after protein coating, the whole material still retained the morphological characteristics of the TNS nanoreticular structure. Figure 1(b) presents the AFM results obtained on the morphological characteristics of the surface of both materials. As shown in Figure 1, the surface of TNS exhibits a uniform nanoprotuberance structure. The nanoprotuberance on the surface of the TNS-LF material on the other hand is more obvious and rounded. Through the analysis of $\mathrm{Ra}$ and $\mathrm{Rz}$ on the surface of the two materials (Table 1), we found that both Ra and Rz of TNS-LF were slightly increased compared with TNS. Results of chemical element analysis on the surface of the 2 materials showed that the content of N, O, and C on the surface of TNS-LF was higher than that of TNS. Especially, the content of the N element increased from almost 0 to about $20 \%$. Figure 1(c) illustrates the result of FTIR analysis. Both kinds of materials were tested and analyzed in the range of $350-4000 \mathrm{~cm}^{-1}$. We found that there were different waveforms in the abscissa coordinate of around $2000 \mathrm{~cm}^{-1}$. After further analysis in the range of $1350-1750 \mathrm{~cm}^{-1}$, it was noted that there were obvious differences between the 2 materials. 

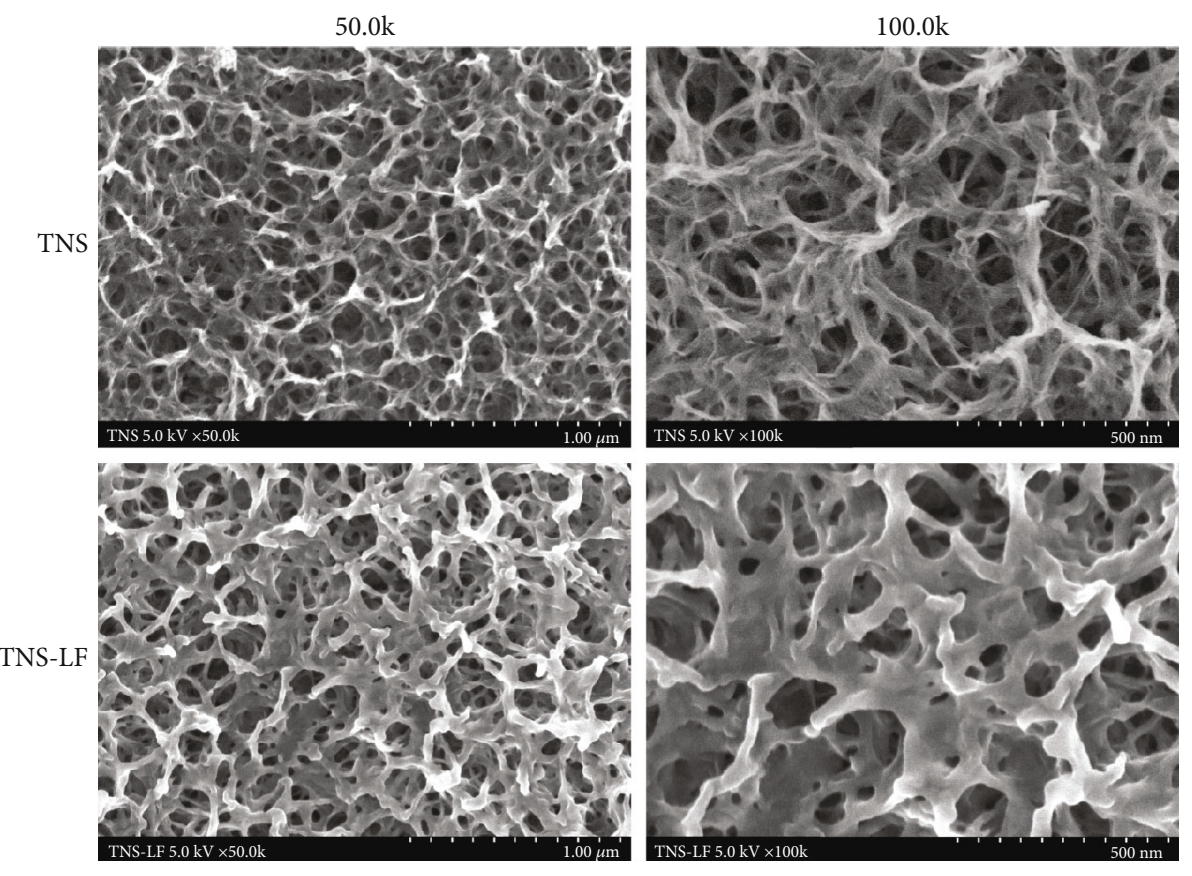

(a)

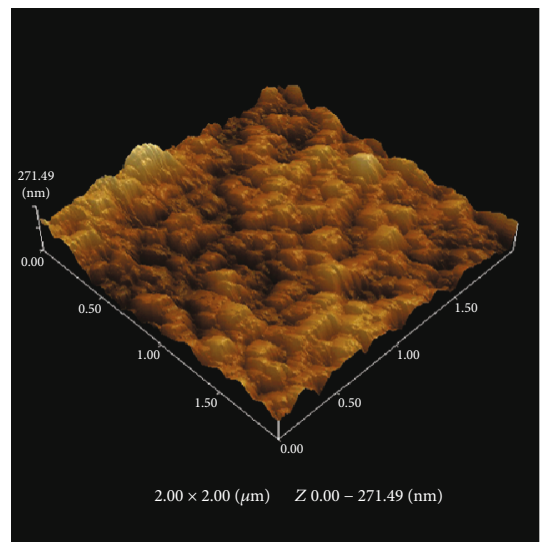

TNS
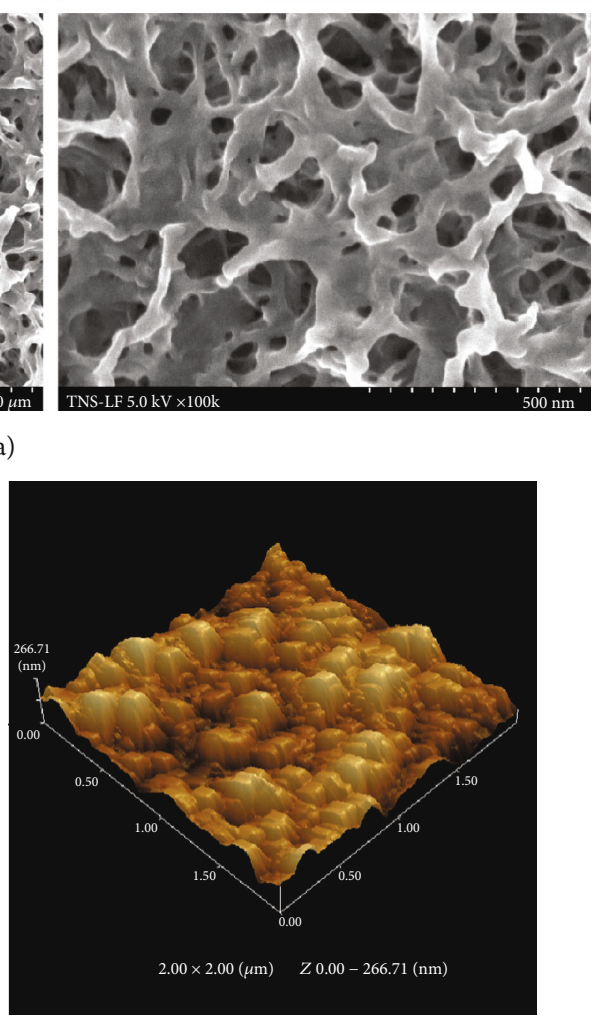

TNS-LF

(b)
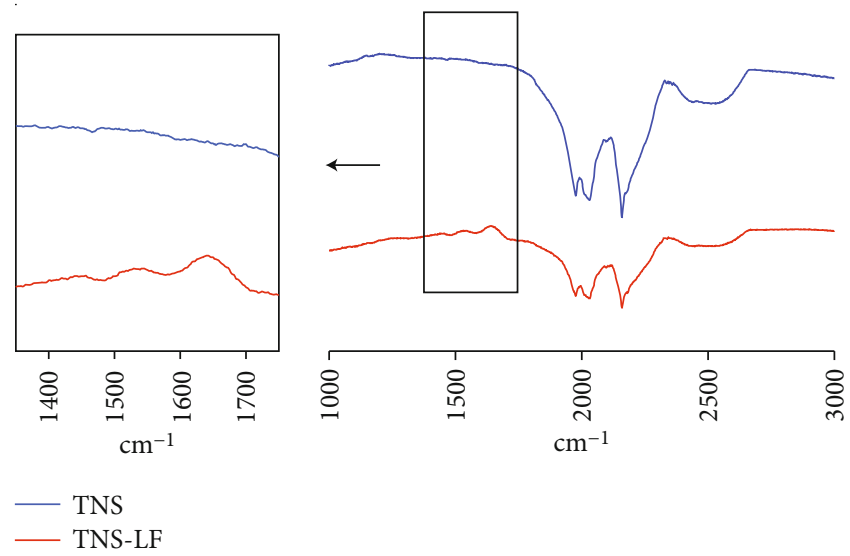

(c)

FIGURE 1: Characterization of the surfaces of TNS and TNS-LF: (a) SEM micrograph images of TNS and TNS-LF; (b) AFM images of TNS and TNS-LF; (c) FTIR analysis of TNS and TNS-LF. TNS: titanium with nanonetwork structures; TNS-LF: titanium with nanonetwork structures coated with lactoferrin. 
TABLE 1: Roughness values of the three different surfaces.

\begin{tabular}{lcc}
\hline Groups & \multicolumn{2}{c}{ Parameters } \\
& $\mathrm{Ra}(\mathrm{nm})$ & $\mathrm{Rz}(\mathrm{nm})$ \\
\hline TNS & $26.45 \pm 2.23$ & $239.57 \pm 26.98$ \\
TNS-LF & $32.07 \pm 2.05$ & $244.51 \pm 18.69$ \\
\hline
\end{tabular}

Ra: mean average surface roughness; Rz: mean peak-to-valley height; TNS: titanium with nanonetwork structures; TNS-LF: titanium with nanonetwork structures coated with lactoferrin.

TABLE 2: Surface chemical compositions of specimens examined by XPS.

\begin{tabular}{lcccc}
\hline Groups & \multicolumn{4}{c}{ Elements } \\
& C1s & N1s & O1s & Ti2p \\
\hline TNS & $18.22 \pm 0.90$ & $0.29 \pm 0.33$ & $58.87 \pm 0.57$ & $22.63 \pm 0.67$ \\
TNS-LF & $66.37 \pm 0.99$ & $14.27 \pm 2.41$ & $22.10 \pm 2.57$ & $0.27 \pm 0.46$ \\
\hline
\end{tabular}

XPS: X-ray photoelectron spectroscopy; TNS-LF: titanium with nanonetwork structures coated with lactoferrin; TNS: titanium with nanonetwork structures.

3.2. Antibacterial Properties. Results of experiments evaluating the antimicrobial properties of samples are shown in Figure 2. Figure 2 (a) shows that the $24 \mathrm{~h}$ antimicrobial rate of TNS-LF was $99 \%$, indicating a remarkable antimicrobial effect of TNS after coating with LF. Similarly, results of the $24 \mathrm{~h}$ biofilm formation experiment (Figure 2(b)) also showed that biofilm formation on the surface of TNS-LF was less apparent than that on the surface of the TNS material. Results of Live/Dead and ROS staining for 1 and $6 \mathrm{~h}$ incubations are shown in Figure 2(c). Results following incubation for $1 \mathrm{~h}$ showed that the number of bacteria adhering to the surface of both materials was relatively low, and more specifically, the amount of bacteria adhering to the surface of the TNS-LF material was significantly $(P<0.001)$ less than that of TNS, and there were more dead bacteria present on TNS-LF than on the TNS material. After incubation for $6 \mathrm{~h}$, bacteria that attached to the surface of the TNS material multiplied in large numbers, whereas the number of bacteria on the surface of the TNS-LF material was increased compared with that after $1 \mathrm{~h}$ incubation, but was still far lower than that of the TNS group. Regarding the results of ROS evaluation, no obvious ROS staining spots were observed in the TNS group at both 1 and $6 \mathrm{~h}$, whereas obvious ROS staining spots could be observed at both time points, on the surface of the TNS-LF material.

\subsection{Evaluation of Osteogenic Induction Ability In Vitro}

3.3.1. Cell Adhesion and Morphology. Figure 3(a) and Figure 4(a) illustrate the initial attachment of rBMMSCs and hPLCs on the surface of each material, respectively. This result indicated that TNS-LF could promote cell attachment on the surface of the material better than TNS at 1, 3, 6, and $24 \mathrm{~h}$, thus providing a basis for cell proliferation and differentiation. Figure 4(b) presents the cell morphology of hPLCs on the surface of both materials at 3 and $6 \mathrm{~h}$. We could clearly observe that hPLCs on the surface of TNS-LF displayed obvious advantages over TNS in both quantity and cell extension.

3.3.2. Evaluation of Osteogenic Induction Ability In Vitro. As shown in Figure 3(b) and Figure 4(c), TNS-LF was able to promote alkaline phosphatase activity, $\mathrm{Ca}$ deposition in extracellular matrix, and osteocalcin (OCN) expression in both cells better than TNS materials.

As shown in Figure 3(b), regarding the expression levels of osteogenic differentiation-related genes, the experimental group (TNS-LF) material demonstrated a significant promoting effect on the expression levels of these genes in rBMMSCs at various observation points compared with the control group (TNS) material.

3.4. Evaluation of Osteogenic Induction Ability In Vivo. Results obtained from the in vivo experiments and their respective data analyses are shown in Figure 5. In the 3D image analysis of Figure 5(a), it can be clearly seen that the new bone that formed around the TNS-LF implant is greater than that around the TNS implant (implant in red, cortical bone in blue, and new bone in Kelly green). Concerning the quality of new bone, analysis of the BV/TV $(P<0.001)$, Tb.N $(P<0.05)$, and Tb.Th $(P<0.001)$ parameters of the new bone that formed around the implant revealed that they were significantly higher in the experimental group compared to the control group.

As shown in Figure 5(b), the area of new bone tissue that was generated around the implants in the experimental group was much larger than that in the control group. After further analysis and calculation of BIC and BA, it was found that the area of new bone around the TNS-LF implant was about 3 times greater than that of TNS, and BIC increased by nearly $20 \%$ compared with TNS.

Results of fluorescence staining visualized by fluorescence confocal microscopy are presented in Figure 5(c). New bone formation around TNS-LF implants increased significantly $(P<0.001 ; P<0.01)$ compared with that around TNS. After calculating the new bone mass generated at 1,4 , and 8 weeks, it was revealed that the new bone that formed around the experimental group was slightly more than that of the control group at week 1 , while the main time period of bone formation was between 4 and 8 weeks, during which period, new bone formation reached about $60 \%$ of the total. Additionally, the amount of new bone generated in the experimental group was greater than that in the control group, at each calculation time point.

\section{Discussion}

In this study, we analyzed the surface characteristics of TNS and TNS-LF materials in detail, evaluated the antimicrobial properties of the 2 materials using Staphylococcus aureus as a model organism, and analyzed the early attachment of rBMMSCs and hPLCs growing on the surface of these materials, as well as the expression levels of markers related to osteogenic differentiation. Concomitantly, animal experiments were also carried out to study the biological properties 


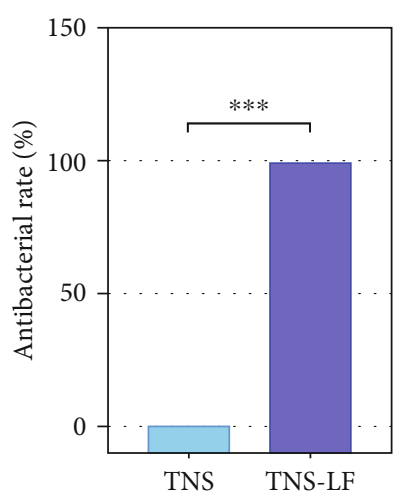

TNS

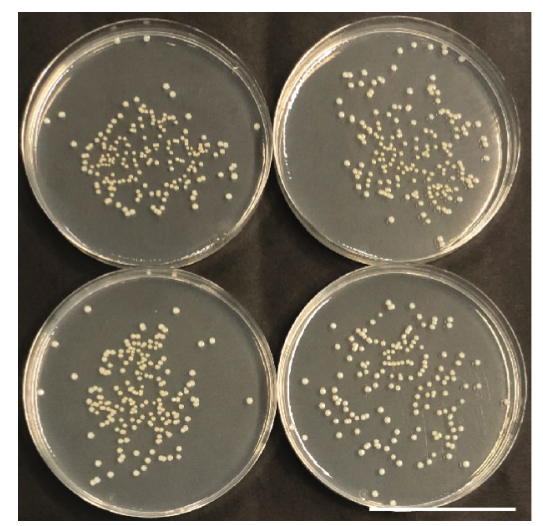

(a)

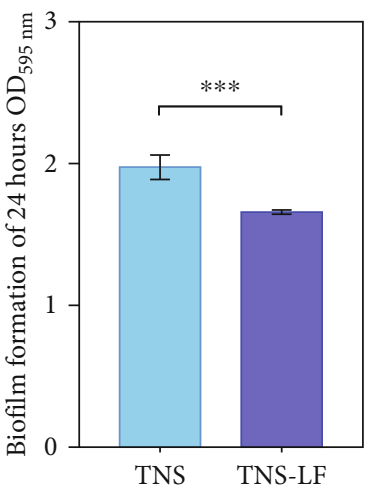

(b)

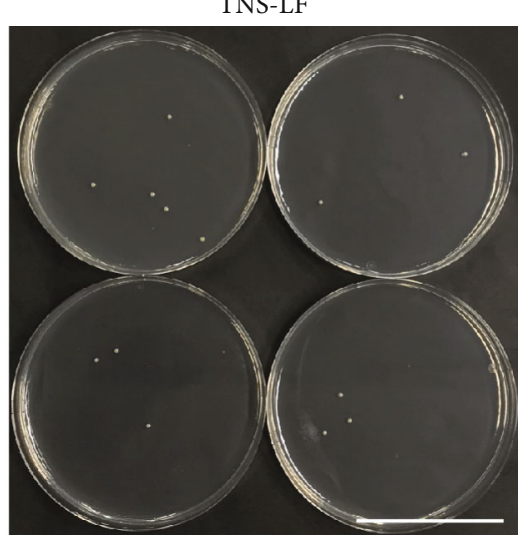

TNS-LF

ROS

Live/Dead
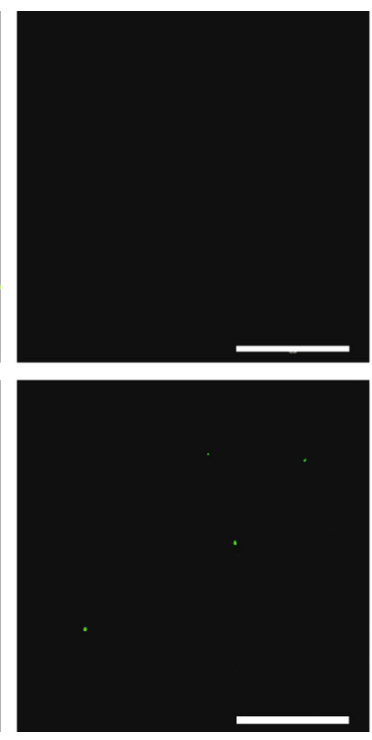
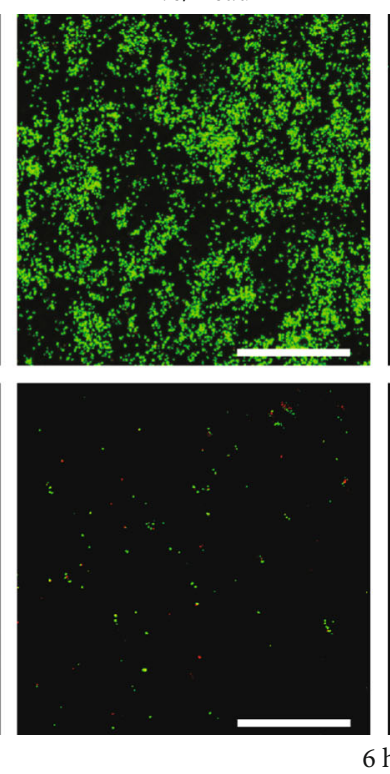

ROS
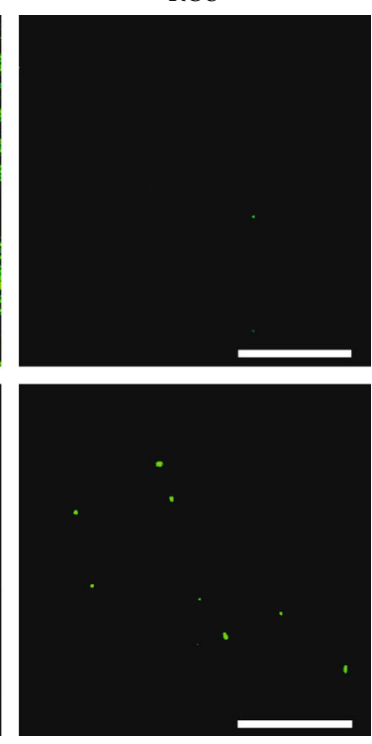

(c)

Figure 2: Antibacterial properties of TNS and TNS-LF: (a) antibacterial rate (scale bar $=5 \mathrm{~cm}$ ); (b) biofilm formation; (c) live/dead staining and ROS of bacteria attached to sample discs (scale bar $=200 \mu \mathrm{m}$ ). ${ }^{* * *} P<0.001$. TNS: titanium with nanonetwork structures; TNS-LF: titanium with nanonetwork structures coated with lactoferrin; ROS: reactive oxygen species. 


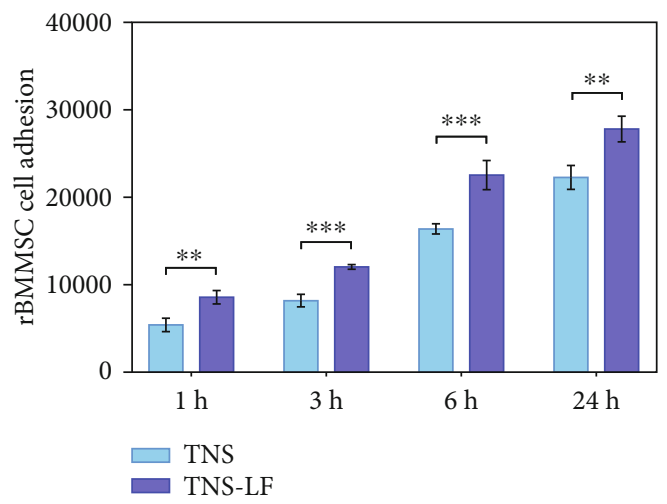

(a)
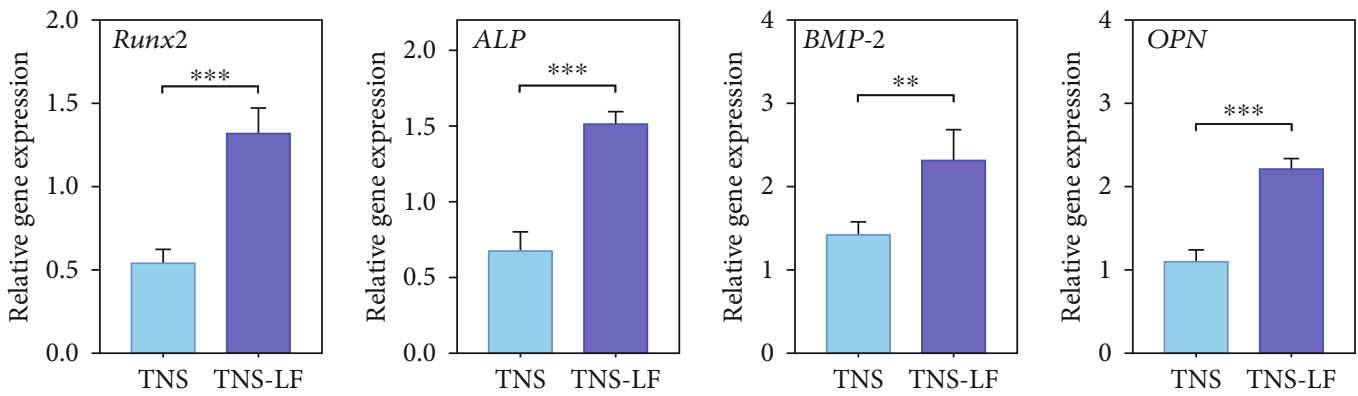

(b)
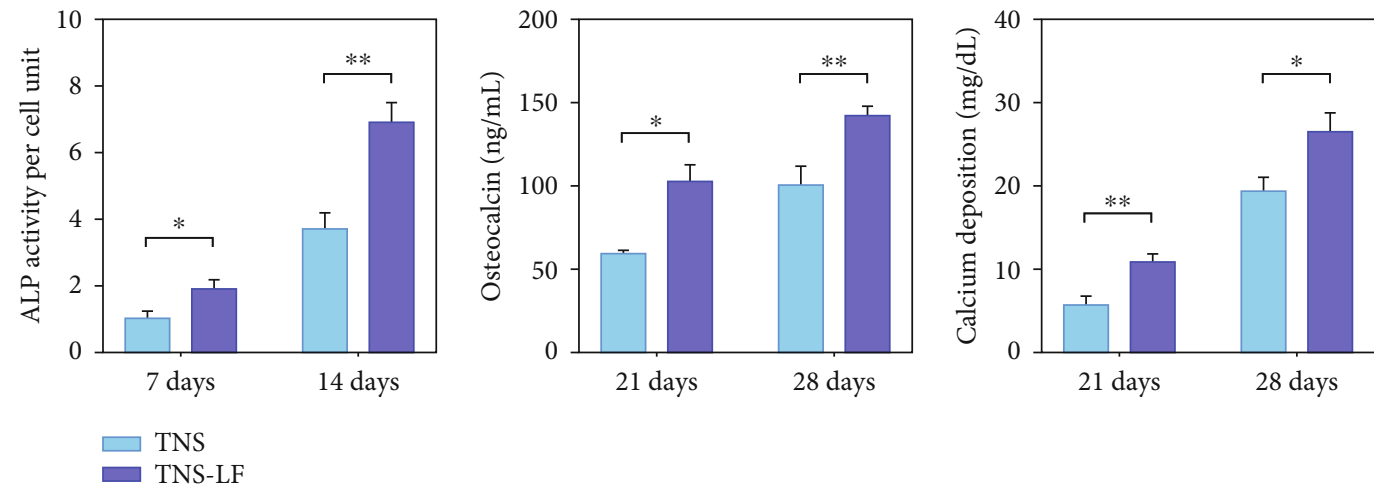

(c)

FIgURE 3: Evaluation of in vitro osteogenic induction ability of rBMMSCs: (a) cell adhesion of rBMMSCs on sample disks; (b) expression of osteogenesis-related genes in cells grown on sample disks; (c) ALP activity, calcium deposition, and production of OCN in cells grown on sample disks. ${ }^{* * *} P<0.001 ;{ }^{* *} P<0.01 ;{ }^{*} P<0.05$. TNS: titanium with nanonetwork structures; TNS-LF: titanium with nanonetwork structures coated with lactoferrin; rBMMSCs: rat bone marrow mesenchymal stem cells; Runx2: runt-related transcription factor 2; ALP: alkaline phosphatase; $B M P$-2:bone morphogenetic protein 2; OPN: osteopontin; OCN: osteocalcin.

of both kinds of implant materials. Our experimental results showed that LF was successfully coated on the surface of TNS, forming a new implant material termed TNS-LF. Compared with TNS, this new material not only exhibited obvious antimicrobial properties but also further promoted cell attachment. Additionally, it was shown to promote the osteogenic differentiation of cells, as demonstrated in animal experiments.

All these findings of characterization of the surfaces showed us that the surface of the TNS material following LF coating exhibits a new chemical form [36, 37]. Results obtained by SEM and AFM investigation showed that alkali treatment at room temperature resulted in a uniform nano- scale network structure on the surface of pure titanium, as was previously described [7, 38]. At the same time, we noticed that LF was uniformly adhered to the surface of the TNS material, but still retained the nanoreticular structure of the original substrate material. Chemical and physiochemical (XPS and FITR) characterization further validated the successful coating of the TNS surface with LF. The reason for choosing TNS as the base material in this study was that according to the results of Quartz Crystal Microbalance Sensor (QCM) analysis of TNS and Ti materials performed in previous studies, we knew that TNS adsorbs proteins more easily than $\mathrm{Ti}[14,39]$. Concurrently, TNS has been shown to display increased hydrophilicity [7] relative to $\mathrm{Ti}$ 


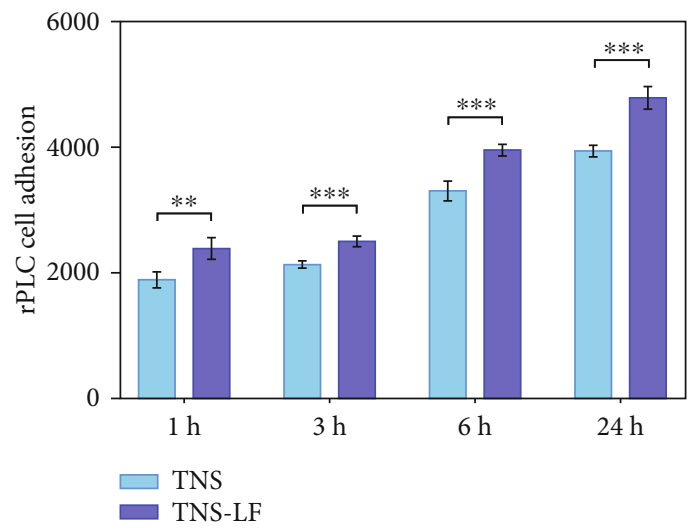

(a)

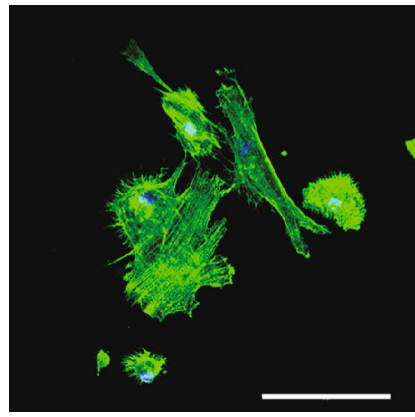

$3 \mathrm{~h}$ TNS

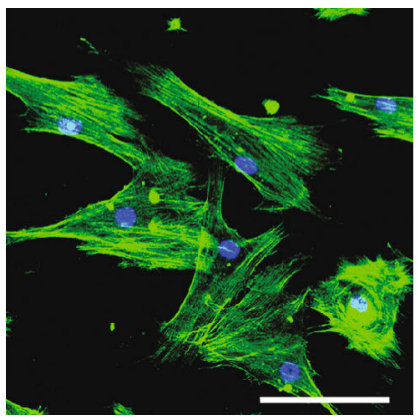

$3 \mathrm{~h}$ TNS-LF

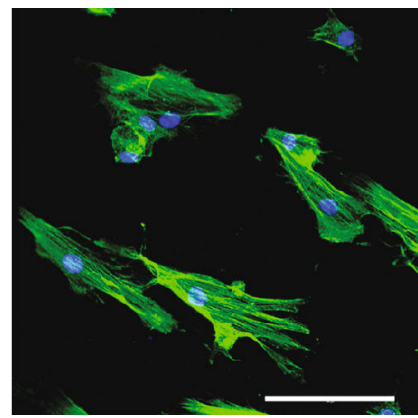

$6 \mathrm{~h} \mathrm{TNS}$

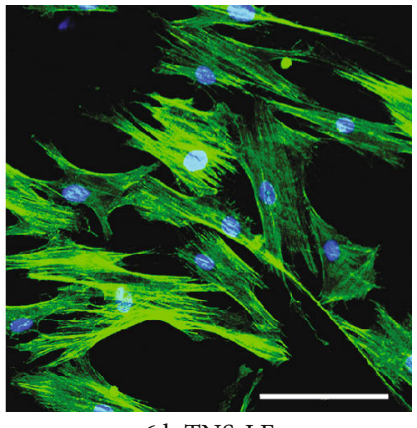

$6 \mathrm{~h}$ TNS-LF

(b)
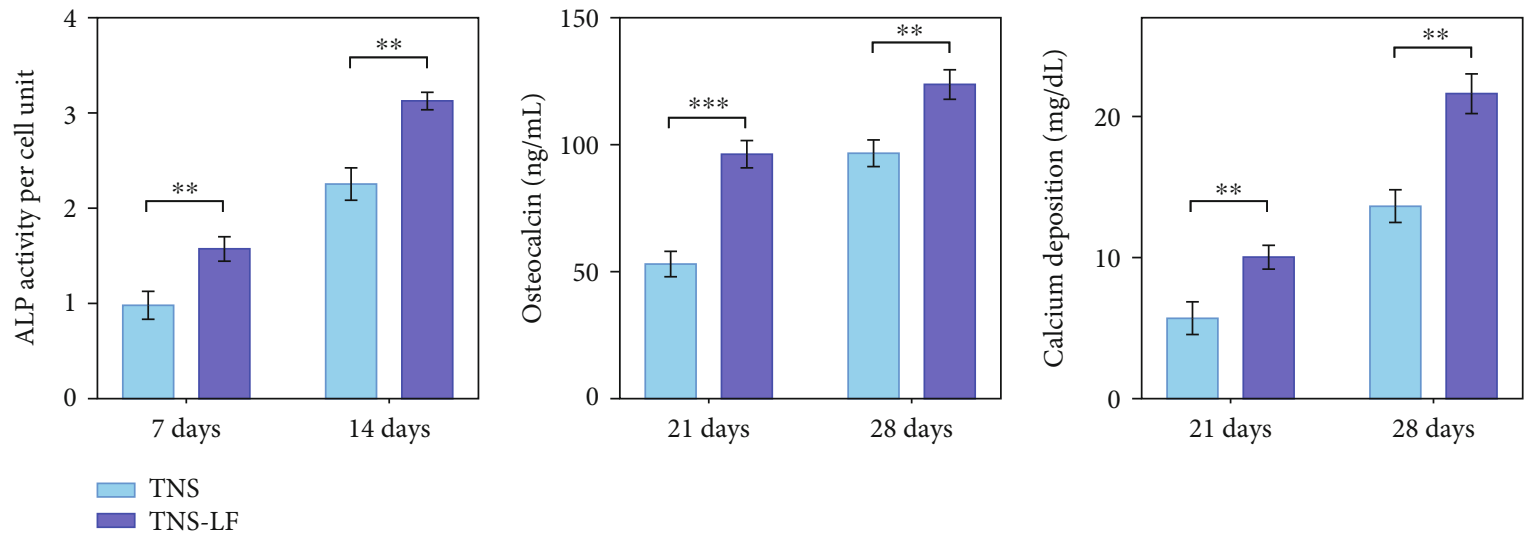

(c)

FIGURE 4: Evaluation of in vitro osteogenic induction ability of rPLCs: (a) cell adhesion of rPLCs on sample disks; (b) morphological analysis of rPLCs on sample disks (scale bar $=100 \mu \mathrm{m}$ ); (c) ALP activity, calcium deposition, and production of OCN in cells grown on sample disks. ${ }^{* * *} P<0.001 ;{ }^{* *} P<0.01$. TNS: titanium with nanonetwork structures; TNS-LF: titanium with nanonetwork structures coated with lactoferrin; rPLCs: rat periodontal ligament cells; ALP: analysis of alkaline phosphatase; OCN: osteocalcin.

materials, allowing the use of a simpler treatment formula. Because there is no interface between the nanoreticular structure layer on the surface of TNS and Ti, the method used can efficiently coat proteins on the surface of TNS, offering a strong binding. Another reason was that our previous studies had concluded that this biocomposite implant was not only a protein-mechanical and TNS-physical-chemical structure, but also a functional combination of the $2[7,14]$. Moreover, no significant differences in the surface roughness of the TNS and TNS-LF surfaces were found.
A serious problem in the clinical setting stems from the fact that microorganisms adhere to abiotic surfaces and form biofilms. In implantation surgery, if a new implant material used could ensure reduced bacterial attachment, it would achieve certain clinical and commercial success, which is of great significance $[4,40]$. In this study, TNS-LF showed more effective antimicrobial properties relative to TNS, which is essential for long-term survival and implant success. Since the TNS material does not have antimicrobial properties $[15,16]$, the antimicrobial properties exhibited by TNS-LF 


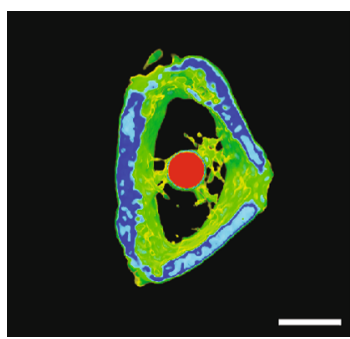

TNS

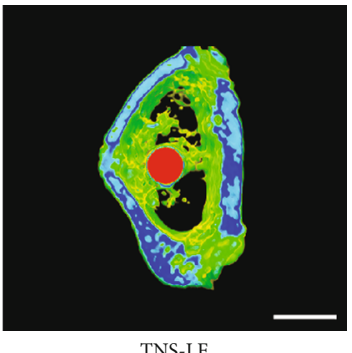

TNS-LF

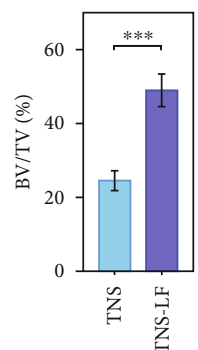

(a)

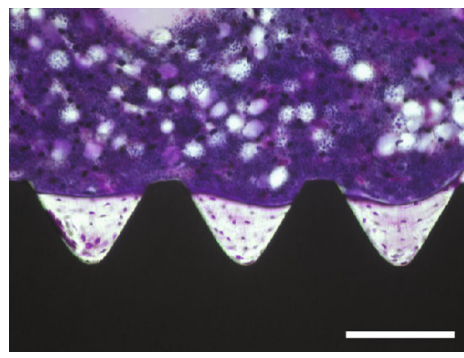

TNS

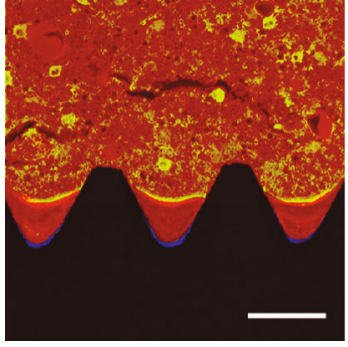

TNS

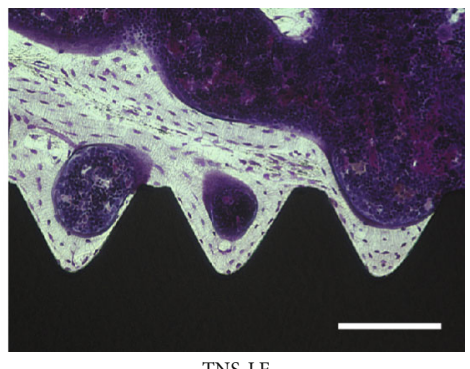

(b)
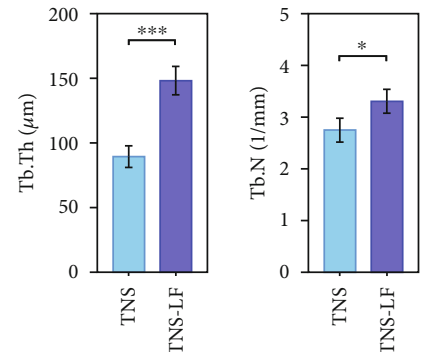
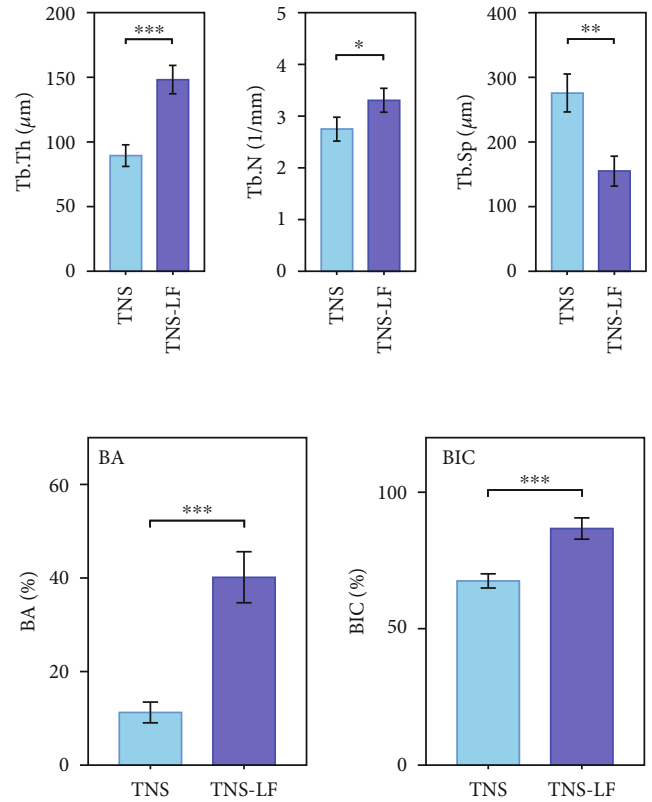

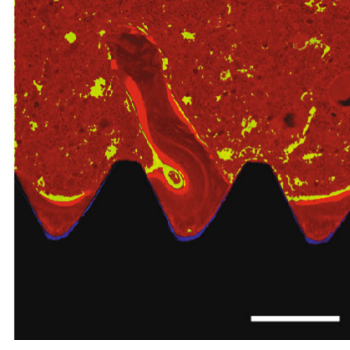

TNS-LF
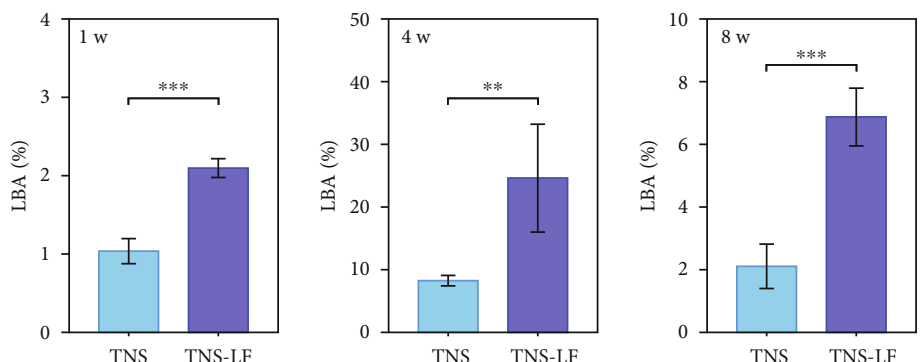

(c)

FIGURE 5: Evaluation of osteogenic induction ability in vivo: (a) transverse micro-CT reconstructed images of proximal tibiae showing ROI status and micro-CT quantitative evaluation within the ROI (scale bar $=2 \mathrm{~mm}$ ); (b) histological sections with Villanueva staining showing bone tissue morphology around the implant (black) and quantitative histomorphometric analysis within the region of measurement (BA and BIC) (scale bar $=200 \mu \mathrm{m}$ ); (c) merged fluorescent images of alloy implants and fluorescence labeling analysis of new bone formation at 1,4 , and 8 weeks after implantation (scale bar $=100 \mu \mathrm{m}$ ). ${ }^{* * *} P<0.001 ;{ }^{* *} P<0.01 ;{ }^{*} P<0.05$. Micro-CT: computed tomography; TNS: titanium with nanonetwork structures; TNS-LF: titanium with nanonetwork structures coated with lactoferrin; ROI: region of interest; BV/TV: bone volume/total volume; Tb.Th: trabecular thickness; Tb.N: trabecular number; Tb.Sp: trabecular separation; BA: bone area ratio; BIC: bone-implant contact; LBA: labeled bone area.

were due to LF coated on the surface of the TNS material. By performing experiments evaluating the in vitro antimicrobial properties of samples, we found that the LF coating provided obvious antibacterial attachment to the sample surface and also exhibited a certain bactericidal effect. After the initial discovery of the antimicrobial activity of LF [41, 42], scholars have demonstrated that LF is a broad-spectrum antimicrobial activity protein and thus can inhibit the growth of many bacteria [43-46] through a direct action on bacteria themselves. It has been reported that the molecular mechanism of the bactericidal effect of LF on Staphylococcus aureus (Gram-positive bacteria) used in this study is likely to be similar to that of cationic and amphiphilic antimicrobial peptides [41]. Antimicrobial peptides bind to Gram-positive bacteria through electrostatic interaction between the negatively charged lipid matrix and cationic amino acid residues in the target membrane. After binding, due to hydrophobic interaction, the parent residues interfere with the interior of the nonpolar membrane $[47,48]$. This seems to correspond to the same $\mathrm{N}$-terminal region of LF, where hydrophobic residues are located close to cationic residues, and amphiphilic cationic titanium obtained at the N-terminal of human or bovine LF has been shown to be many times more active to bacteria than the parent protein itself [41]. Likewise, it has been reported that LF can be used as an inhibitor of bacterial biofilm formation under certain conditions [49-51].

From the results obtained in this study, it could be clearly suggested that TNS-LF materials are able to promote the early attachment of cells and promote the osteogenic differentiation of rBMMSCs. The amount of new bone formed around TNS-LF implants and the implant bone binding rate in the observation area were higher than those in the control 
group. It has been reported that the mechanism of LF action in osteoblasts might be due to the mitogenic effect shown in osteoblasts, mainly mediated by LRP1 and related to the activation of IGF1, Ptgs2, and Nfatc1. LF-induced activation of p42/44 MAPK and PI3-kinase-dependent ATP signaling in osteoblasts is also another possibility [28]. Successful implant-bone bonding requires the concerted efforts of different types of cells, which should not be ignored in some cases of immediate implantation after tooth extraction. For example, the periodontal ligament plays a certain physiological function by attaching to the cementum and alveolar bone. It has been reported that there are still periodontal ligament cells attached to the side of the alveolar bone in the extraction socket after tooth extraction. The results of our study showed that the TNS-LF material could not only promote the adhesion and osteogenic differentiation of rBMMSCs around the material but also promote the adhesion of hPLCs and induce osteogenic differentiation, an additional advantageous property of this material.

\section{Conclusions}

We used simple alkali treatment to obtain a stable experimental TNS base material and then applied a simple method to obtain an appropriate concentration of LF coating on the surface of TNS, which could promote the osteogenic differentiation of bone marrow mesenchymal stem cells as well as the osteogenesis of periodontal ligament cells. Concurrently, the LF physiological concentration was not able to exert too much effect on vascular endothelial cells, but was still adequate in hindering formation of osteoclasts, thus providing its positive role in regulating a variety of cell behaviors in the process of bone binding. In addition, TNS-LF also exhibited antimicrobial properties, providing a guarantee for the success of implants. Importantly, the effect was obvious despite of the whole process of material treatment being relatively simple, and the cost of treatment being relatively low. The purpose of this study was to provide a case of such an implant, and to analyze the possibility and value of its clinical use from various perspectives, so as to provide a basis for future experiments. Experiments evaluating the effect of these materials on macrophages, osteoclasts, and vascular endothelial cells need to be further performed, and we will continue to attempt to identify the ideal concentration of this material around physiological concentrations to achieve the best balance between promoting osteogenesis and antimicrobial properties.

\section{Data Availability}

The data used to support the findings of this study are available from the corresponding author upon request.

\section{Additional Points}

Statement of Significance. The purpose of this work is to use simple and inexpensive methods to treat implant materials and integrate biological proteins on the surface to improve the biological properties of materials. Especially, the TNS material coated with LF functions as an antibacterial and promotes osteogenesis. It is noteworthy that this material can also induce osteogenesis of periodontal ligament cells, which provides a new implant material for immediate implantation after tooth extraction. On the basis of other scholars' research and our previous work, we chose the appropriate LF concentration to prove the superiority of this material from the aspects of material surface analysis experiment, cell experiment, and animal experiment, which provides a theoretical possibility for the clinical application of this material.

\section{Conflicts of Interest}

The authors declare that they have no conflicts of interest.

\section{Acknowledgments}

We are grateful to our colleagues for their help and suggestions in this study. It is our privilege to be a member of the Department of Removable Prosthodontics and Occlusion. We also wish to thank Mr. H. Hori and Mr. N. Kawade from the Institute of Dental Research of Osaka Dental University for their kind help with experimental techniques. This work was supported by the Shenzhen Healthcare Research Project (No. SZXJ2017064). In particular, I would like to thank the Osaka Dental University Research Funds (grant number: 19-10) and the Otsuka Toshimi Scholarship Foundation for the support in my life.

\section{References}

[1] D. Buser, L. Sennerby, and H. De Bruyn, "Modern implant dentistry based on osseointegration: 50 years of progress, current trends and open questions," Periodontology 2000, vol. 73, no. 1, pp. 7-21, 2017.

[2] M. M. Bornstein, S. Halbritter, H. Harnisch, H.-P. Weber, and D. Buser, "A retrospective analysis of patients referred for implant placement to a specialty clinic: indications, surgical procedures, and early failures," The International Journal of Oral \& Maxillofacial Implants, vol. 23, no. 6, pp. 1109-1116, 2008.

[3] O. Brügger, M. Bornstein, U. Kuchler, S. Janner, V. Chappuis, and D. Buser, "Implant therapy in a surgical specialty clinic: an analysis of patients, indications, surgical procedures, risk factors, and early failures," The International Journal of Oral \& Maxillofacial Implants, vol. 30, no. 1, pp. 151-160, 2015.

[4] S. Spriano, S. Yamaguchi, F. Baino, and S. Ferraris, "A critical review of multifunctional titanium surfaces: new frontiers for improving osseointegration and host response, avoiding bacteria contamination," Acta Biomaterialia, vol. 79, pp. 1-22, 2018.

[5] E. O’Neill, G. Awale, L. Daneshmandi, O. Umerah, and K. W. H. Lo, "The roles of ions on bone regeneration," Drug Discovery Today, vol. 23, no. 4, pp. 879-890, 2018.

[6] H. Chouirfa, H. Bouloussa, V. Migonney, and C. FalentinDaudré, "Review of titanium surface modification techniques and coatings for antibacterial applications," Acta Biomaterialia, vol. 83, pp. 37-54, 2019.

[7] D. Yin, S. Komasa, S. Yoshimine, T. Sekino, and J. Okazaki, "Effect of mussel adhesive protein coating on osteogenesis 
in vitro and osteointegration in vivo to alkali-treated titanium with nanonetwork structures," International Journal of Nanomedicine, vol. Volume 14, pp. 3831-3843, 2019.

[8] H. M. Kim, F. Miyaji, T. Kokubo, and T. Nakamura, "Preparation of bioactive $\mathrm{Ti}$ and its alloys via simple chemical surface treatment," Journal of Biomedical Materials Research, vol. 32, no. 3, pp. 409-417, 1996.

[9] S. Nishiguchi, H. Kato, H. Fujita et al., "Titanium metals form direct bonding to bone after alkali and heat treatments," Biomaterials, vol. 22, no. 18, pp. 2525-2533, 2001.

[10] H. Xing, S. Komasa, Y. Taguchi, T. Sekino, and J. Okazaki, "Osteogenic activity of titanium surfaces with nanonetwork structures," International Journal of Nanomedicine, p. 1741, 2014.

[11] S. Komasa, Y. Taguchi, H. Nishida, M. Tanaka, and T. Kawazoe, "Bioactivity of nanostructure on titanium surface modified by chemical processing at room temperature," Journal of Prosthodontic Research, vol. 56, no. 3, pp. 170-177, 2012.

[12] T. Kusumoto, D. Yin, H. Zhang et al., "Evaluation of the osteointegration of a novel alkali-treated implant system $<\mathrm{i}>$ In Vivo</i>," Journal of Hard Tissue Biology, vol. 26, no. 4, pp. 355-360, 2017.

[13] L. Chen, S. Komasa, Y. Hashimoto, S. Hontsu, and J. Okazaki, "In vitro and in vivo osteogenic activity of titanium implants coated by pulsed laser deposition with a thin film of fluoridated hydroxyapatite," International Journal of Molecular Sciences, vol. 19, no. 4, p. 1127, 2018.

[14] C. Terada, S. Komasa, T. Kusumoto, T. Kawazoe, and J. Okazaki, "Effect of amelogenin coating of a nano-modified titanium surface on bioactivity," International Journal of Molecular Sciences, vol. 19, no. 5, p. 1274, 2018.

[15] H. Zhang, S. Komasa, C. Mashimo, T. Sekino, and J. Okazaki, "Effect of ultraviolet treatment on bacterial attachment and osteogenic activity to alkali-treated titanium with nanonetwork structures," International Journal of Nanomedicine, vol. Volume 12, pp. 4633-4646, 2017.

[16] H. Zhang, M. Hatoko, D. Yin et al., “Antibacterial Activity and Biocompatibility of Nanoporous Titanium Doped with Silver Nanoparticles and Coated with N-Acetyl Cysteine," Journal of Hard Tissue Biology, vol. 27, no. 4, pp. 351-358, 2018.

[17] Y. Su, S. Komasa, P. Li et al., "Synergistic effect of nanotopography and bioactive ions on peri-implant bone response," International Journal of Nanomedicine, vol. Volume 12, pp. 925-934, 2017.

[18] S. A. González-Chávez, S. Arévalo-Gallegos, and Q. RascónCruz, "Lactoferrin: structure, function and applications," International Journal of Antimicrobial Agents, vol. 33, no. 4, pp. 301.e1-301.e8, 2009.

[19] M. Godoy-Gallardo, C. Mas-Moruno, M. C. Fernández-Calderón et al., "Covalent immobilization of hLf1-11 peptide on a titanium surface reduces bacterial adhesion and biofilm formation," Acta Biomaterialia, vol. 10, no. 8, pp. 35223534, 2014.

[20] H. Jenssen and R. Hancock, "Antimicrobial properties of lactoferrin,” Biochimie, vol. 91, no. 1, pp. 19-29, 2009.

[21] F. Berlutti, F. Pantanella, T. Natalizi et al., "Antiviral properties of lactoferrin-a natural immunity molecule," Molecules, vol. 16, no. 8, pp. 6992-7018, 2011.

[22] A. A. Amini and L. S. Nair, "Lactoferrin: A Biologically Active Molecule for Bone Regeneration," Current Medicinal Chemistry, vol. 18, no. 8, pp. 1220-1229, 2011.
[23] Y. Xu, T. Zhao, H. Ren et al., "Urinary metabolic profiling via lc-ms/ms reveals impact of bovine lactoferrin on bone formation in growing sd rats," Nutrients, vol. 12, no. 4, pp. 11161137, 2020.

[24] R. Gao, M. Watson, K. E. Callon et al., "Local application of lactoferrin promotes bone regeneration in a rat critical-sized calvarial defect model as demonstrated by micro-CT and histological analysis," Journal of Tissue Engineering and Regenerative Medicine, vol. 12, no. 1, pp. e620-e626, 2018.

[25] Y. Xu, J. J. An, D. Tabys et al., "Effect of lactoferrin on the expression profiles of long non-coding RNA during osteogenic differentiation of bone marrow mesenchymal stem cells," International Journal of Molecular Sciences, vol. 20, no. 19, pp. 4834-4852, 2019.

[26] M. Icriverzi, V. Dinca, M. Moisei, R. W. Evans, M. Trif, and A. Roseanu, "Lactoferrin in bone tissue regeneration," Current Medicinal Chemistry, vol. 27, no. 6, pp. 838-853, 2020.

[27] J. H. Brock, "The physiology of lactoferrin," Biochemistry and Cell Biology, vol. 80, no. 1, pp. 1-6, 2002.

[28] J. Cornish and D. Naot, "Lactoferrin as an effector molecule in the skeleton," Biometals, vol. 23, no. 3, pp. 425-430, 2010.

[29] G. Majka, K. Śpiewak, K. Kurpiewska et al., "A highthroughput method for the quantification of iron saturation in lactoferrin preparations," Analytical and Bioanalytical Chemistry, vol. 405, no. 15, pp. 5191-5200, 2013.

[30] J. Cornish, K. Palmano, K. E. Callon et al., "Lactoferrin and bone; structure-activity relationships," Biochemistry and Cell Biology, vol. 84, no. 3, pp. 297-302, 2006.

[31] J. Cornish, K. E. Callon, D. Naot et al., "Lactoferrin is a potent regulator of bone cell activity and increases bone formation in vivo," Endocrinology, vol. 145, no. 9, pp. 4366-4374, 2004.

[32] F. Lorget, J. Clough, M. Oliveira, M. C. Daury, A. Sabokbar, and E. Offord, "Lactoferrin reduces in vitro osteoclast differentiation and resorbing activity," Biochemical and Biophysical Research Communications, vol. 296, no. 2, pp. 261-266, 2002.

[33] J. Lee, J. Lee, S. Lee et al., "Bioactive membrane immobilized with lactoferrin for modulation of bone regeneration and inflammation," Tissue Engineering Part A, 2020.

[34] J. An, Y. Xu, Z. Kong et al., "Effect of lactoferrin and its digests on differentiation activities of bone mesenchymal stem cells," Journal of Functional Foods, vol. 57, pp. 202-210, 2019.

[35] M. Shimamura, Y. Yamamoto, H. Ashino et al., "Bovine lactoferrin inhibits tumor-induced angiogenesis," International Journal of Cancer, vol. 111, no. 1, pp. 111-116, 2004.

[36] C. Constantinescu, A. Palla-Papavlu, A. Rotaru et al., "Multifunctional thin films of lactoferrin for biochemical use deposited by MAPLE technique," Applied Surface Science, vol. 255, no. 10, pp. 5491-5495, 2009.

[37] Y. Joubran, A. Mackie, and U. Lesmes, "Impact of the Maillard reaction on the antioxidant capacity of bovine lactoferrin," Food Chemistry, vol. 141, no. 4, pp. 3796-3802, 2013.

[38] T. Kasuga, M. Hiramatsu, A. Hoson, T. Sekino, and K. Niihara, "Titania nanotubes prepared by chemical processing," Advanced Materials, vol. 11, no. 15, pp. 1307-1311, 1999.

[39] Y. Tashiro, S. Komasa, A. Miyake, H. Nishizaki, and J. Okazaki, "Analysis of titania nanosheet adsorption behavior using a quartz crystal microbalance sensor," Advances in Materials Science and Engineering, vol. 2018, Article ID 7461245, 10 pages, 2018.

[40] Y. Li, Y. Yang, R. Li et al., "Enhanced antibacterial properties of orthopedic implants by titanium nanotube surface 
modification: a review of current techniques," International Journal of Nanomedicine, vol. Volume 14, pp. 7217-7236, 2019.

[41] P. Valenti and G. Antonini, "Lactoferrin: an important host defence against microbial and viral attack," Cellular and Molecular Life Sciences, vol. 62, no. 22, pp. 2576-2587, 2005.

[42] P. L. Masson, J. F. Heremans, J. J. Prignot, and G. Wauters, "Immunohistochemical localization and bacteriostatic properties of an iron-binding protein from bronchial mucus," Thorax, vol. 21, no. 6, pp. 538-544, 1966.

[43] B. Lönnerdal and S. Iyer, "Lactoferrin: Molecular Structure and Biological Function," Annual Review of Nutrition, vol. 15, no. 1, pp. 93-110, 1995.

[44] R. Pakkanen and J. Aalto, "Growth factors and antimicrobial factors of bovine colostrum," International Dairy Journal, vol. 7, no. 5, pp. 285-297, 1997.

[45] E. D. Weinberg, "Human lactoferrin: a novel therapeutic with broad spectrum potential," The Journal of Pharmacy and Pharmacology, vol. 53, no. 10, pp. 1303-1310, 2001.

[46] N.-Y. Lee, K. Kawai, I. Nakamura, T. Tanaka, H. Kumura, and K.-i. Shimazaki, "Susceptibilities against Bovine Lactoferrin with Microorganisms Isolated from Mastitic Milk," Journal of Veterinary Medical Science, vol. 66, no. 10, pp. 1267-1269, 2004.

[47] A. Tossi, L. Sandri, and A. Giangaspero, "Amphipathic, alphahelical antimicrobial peptides,” Biopolymers, vol. 55, no. 1, pp. 4-30, 2000.

[48] A. Wessolowski, M. Bienert, and M. Dathe, "Antimicrobial activity of arginine-and tryptophan-rich hexapeptides: the effects of aromatic clusters, D-amino acid substitution and cyclization," The Journal of Peptide Research, vol. 64, no. 4, pp. 159-169, 2004.

[49] M. Tomita, W. Bellamy, M. Takase, K. Yamauchi, H. Wakabayashi, and K. Kawase, "Potent antibacterial peptides generated by pepsin digestion of bovine lactoferrin," Journal of Dairy Science, vol. 74, no. 12, pp. 4137-4142, 1991.

[50] W. Bellamy, M. Takase, H. Wakabayashi, K. Kawase, and M. Tomita, "Antibacterial spectrum of lactoferricin B, a potent bactericidal peptide derived from the $\mathrm{N}$-terminal region of bovine lactoferrin," The Journal of Applied Bacteriology, vol. 73, no. 6, pp. 472-479, 1992.

[51] K. Yamauchi, M. Tomita, T. J. Giehl, and R. T. Ellison 3rd, "Antibacterial activity of lactoferrin and a pepsin-derived lactoferrin peptide fragment," Infection and Immunity, vol. 61, no. 2, pp. 719-728, 1993. 